\title{
THE MUON ANOMALOUS MAGNETIC MOMENT
}

Updated August 2013 by A. Hoecker (CERN), and W.J. Marciano (BNL).

The Dirac equation predicts a muon magnetic moment, $\vec{M}=g_{\mu} \frac{e}{2 m_{\mu}} \vec{S}$, with gyromagnetic ratio $g_{\mu}=2$. Quantum loop effects lead to a small calculable deviation from $g_{\mu}=2$, parameterized by the anomalous magnetic moment

$$
a_{\mu} \equiv \frac{g_{\mu}-2}{2} .
$$

That quantity can be accurately measured and, within the Standard Model (SM) framework, precisely predicted. Hence, comparison of experiment and theory tests the SM at its quantum loop level. A deviation in $a_{\mu}^{\exp }$ from the SM expectation would signal effects of new physics, with current sensitivity reaching up to mass scales of $\mathcal{O}(\mathrm{TeV})[1,2]$. For recent and very thorough muon $g-2$ reviews, see Refs. [3-5].

The E821 experiment at Brookhaven National Lab (BNL) studied the precession of $\mu^{+}$and $\mu^{-}$in a constant external magnetic field as they circulated in a confining storage ring. It found $[7]^{1}$

$$
\begin{aligned}
& a_{\mu+}^{\exp }=11659204(6)(5) \times 10^{-10}, \\
& a_{\mu-}^{\exp }=11659215(8)(3) \times 10^{-10},
\end{aligned}
$$

where the first errors are statistical and the second systematic. Assuming CPT invariance and taking into account correlations between systematic uncertainties, one finds for their average $[6,7]$

$$
a_{\mu}^{\exp }=11659209.1(5.4)(3.3) \times 10^{-10} .
$$

These results represent about a factor of 14 improvement over the classic CERN experiments of the 1970's [8]. Improvement

1 The original results reported by the experiment have been updated in Eq. (2) and Eq. (3) to the newest value for the absolute muon-to-proton magnetic ratio $\lambda=3.183345107(84)$ [6]. The change induced in $a_{\mu}^{\exp }$ with respect to the value of $\lambda=$ 3.18334539 (10) used in Ref. 7 amounts to $+1.12 \times 10^{-10}$. 

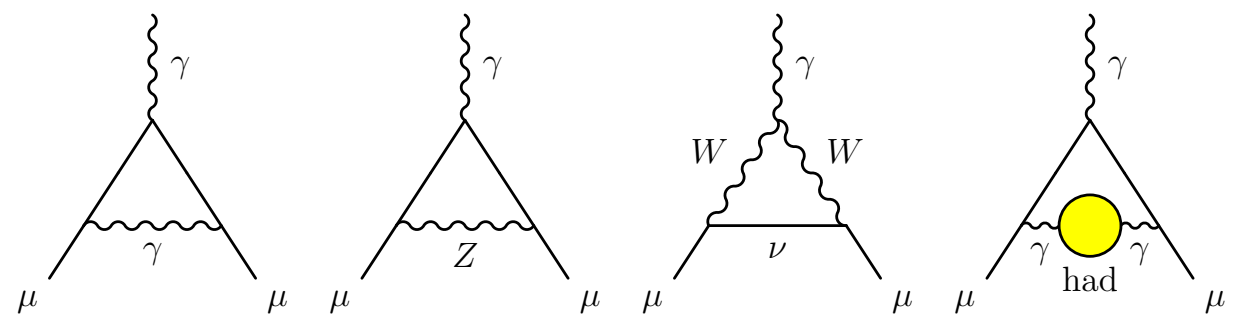

Figure 1: Representative diagrams contributing to $a_{\mu}^{\mathrm{SM}}$. From left to right: first order QED (Schwinger term), lowest-order weak, lowestorder hadronic.

of the measurement in Eq. (3) by a factor of four by moving the E821 storage ring to Fermilab, and utilizing a cleaner and more intense muon beam is in progress. An even more ambitious precision goal is set by an experiment based on a beam of ultra-cold muons proposed at the Japan Proton Accelerator Research Complex.

The SM prediction for $a_{\mu}^{\mathrm{SM}}$ is generally divided into three parts (see Fig. 1 for representative Feynman diagrams)

$$
a_{\mu}^{\mathrm{SM}}=a_{\mu}^{\mathrm{QED}}+a_{\mu}^{\mathrm{EW}}+a_{\mu}^{\mathrm{Had}} .
$$

The QED part includes all photonic and leptonic $(e, \mu, \tau)$ loops starting with the classic $\alpha / 2 \pi$ Schwinger contribution. It has been computed through 5 loops [9]

$$
\begin{aligned}
a_{\mu}^{\mathrm{QED}}= & \frac{\alpha}{2 \pi}+0.765857425(17)\left(\frac{\alpha}{\pi}\right)^{2}+24.05050996(32)\left(\frac{\alpha}{\pi}\right)^{3} \\
& +130.8796(63)\left(\frac{\alpha}{\pi}\right)^{4}+753.3(1.0)\left(\frac{\alpha}{\pi}\right)^{5}+\cdots
\end{aligned}
$$

with a few significant changes in the coefficients since our previous update of this review in 2011. Employing ${ }^{2} \alpha^{-1}=$ 137.035999049(90), obtained [6] from the precise measurements of $h / m_{\mathrm{Rb}}$ [11], the Rydberg constant and $m_{\mathrm{Rb}} / m_{e}$ [6], leads to $[9]$

$$
a_{\mu}^{\mathrm{QED}}=116584718.95(0.08) \times 10^{-11}
$$

2 In the previous versions of this review we used the precise $\alpha$ value determined from the electron $a_{e}$ measurement $[9,10]$. With the new measurement [11] of the recoil velocity of Rubidium, $h / m_{\mathrm{Rb}}$, an $a_{e}$-independent determination of $\alpha$ with sufficient precision is available and preferred. 
where the small error results mainly from the uncertainty in $\alpha$.

Loop contributions involving heavy $W^{ \pm}, Z$ or Higgs particles are collectively labeled as $a_{\mu}^{\mathrm{EW}}$. They are suppressed by at least a factor of $\frac{\alpha}{\pi} \frac{m_{\mu}^{2}}{m_{W}^{2}} \simeq 4 \times 10^{-9}$. At 1-loop order [12]

$$
\begin{aligned}
a_{\mu}^{\mathrm{EW}}[1 \text { loop }]= & \frac{G_{\mu} m_{\mu}^{2}}{8 \sqrt{2} \pi^{2}}\left[\frac{5}{3}+\frac{1}{3}\left(1-4 \sin ^{2} \theta_{\mathrm{W}}\right)^{2}\right. \\
& \left.+\mathcal{O}\left(\frac{m_{\mu}^{2}}{M_{W}^{2}}\right)+\mathcal{O}\left(\frac{m_{\mu}^{2}}{m_{H}^{2}}\right)\right] \\
= & 194.8 \times 10^{-11}
\end{aligned}
$$

for $\sin ^{2} \theta_{\mathrm{W}} \equiv 1-M_{W}^{2} / M_{Z}^{2} \simeq 0.223$, and where $G_{\mu} \simeq 1.166 \times$ $10^{-5} \mathrm{GeV}^{-2}$ is the Fermi coupling constant. Two-loop corrections are relatively large and negative [13]. For a Higgs boson mass of $\simeq 126 \mathrm{GeV}[13]$

$$
a_{\mu}^{\mathrm{EW}}[2-\mathrm{loop}]=-41.2(1.0) \times 10^{-11}
$$

where the uncertainty stems from quark triangle loops. The 3-loop leading logarithms are negligible [13,14], $\mathcal{O}\left(10^{-12}\right)$, implying in total

$$
a_{\mu}^{\mathrm{EW}}=153.6(1.0) \times 10^{-11}
$$

Hadronic (quark and gluon) loop contributions to $a_{\mu}^{\mathrm{SM}}$ give rise to its main theoretical uncertainties. At present, those effects are not calculable from first principles, but such an approach, at least partially, may become possible as lattice QCD matures. Instead, one currently relies on a dispersion relation approach to evaluate the lowest-order (i.e., $\mathcal{O}\left(\alpha^{2}\right)$ ) hadronic vacuum polarization contribution $a_{\mu}^{\mathrm{Had}}[\mathrm{LO}]$ from corresponding cross section measurements [15]

$$
a_{\mu}^{\mathrm{Had}}[\mathrm{LO}]=\frac{1}{3}\left(\frac{\alpha}{\pi}\right)^{2} \int_{m_{\pi}^{2}}^{\infty} d s \frac{K(s)}{s} R^{(0)}(s)
$$


where $K(s)$ is a QED kernel function [16], and where $R^{(0)}(s)$ denotes the ratio of the bare ${ }^{3}$ cross section for $e^{+} e^{-}$annihilation into hadrons to the pointlike muon-pair cross section at centerof-mass energy $\sqrt{s}$. The function $K(s) \sim 1 / s$ in Eq. (10) gives a strong weight to the low-energy part of the integral. Hence, $a_{\mu}^{\mathrm{Had}}[\mathrm{LO}]$ is dominated by the $\rho(770)$ resonance.

Currently, the available $\sigma\left(e^{+} e^{-} \rightarrow\right.$ hadrons) data give a leading-order hadronic vacuum polarization (representative) contribution of [17]

$$
a_{\mu}^{\mathrm{Had}}[\mathrm{LO}]=6923(42)(3) \times 10^{-11},
$$

where the first error is experimental (dominated by systematic uncertainties), and the second due to perturbative QCD, which is used at intermediate and large energies to predict the contribution from the quark-antiquark continuum. New multihadron data from the BABAR experiment have increased the constraints on unmeasured exclusive final states and led to a small reduction in the hadronic contribution compared to the 2009 PDG value.

Alternatively, one can use precise vector spectral functions from $\tau \rightarrow \nu_{\tau}+$ hadrons decays [18] that can be related to isovector $e^{+} e^{-} \rightarrow$ hadrons cross sections by isospin symmetry. Replacing $e^{+} e^{-}$data in the two-pion and four-pion channels by the corresponding isospin-transformed $\tau$ data, and applying isospin-violating corrections (from QED and $m_{d}-m_{u} \neq 0$ ), one finds $[17]$

$$
a_{\mu}^{\text {Had }}[\mathrm{LO}]=7015(42)(19)(3) \times 10^{-11}(\tau),
$$

where the first error is experimental, the second estimates the uncertainty in the isospin-breaking corrections applied to the $\tau$ data, and the third error is due to perturbative QCD. The

3 The bare cross section is defined as the measured cross section corrected for initial-state radiation, electron-vertex loop contributions and vacuum-polarization effects in the photon propagator. However, QED effects in the hadron vertex and final state, as photon radiation, are included. 
current discrepancy between the $e^{+} e^{-}$and $\tau$-based determinations of $a_{\mu}^{\mathrm{Had}}[\mathrm{LO}]$ has been reduced to $1.8 \sigma$ with respect to earlier evaluations. New $e^{+} e^{-}$and $\tau$ data from the $B$-factory experiments BABAR and Belle have increased the experimental information. Reevaluated isospin-breaking corrections have also contributed to this improvement [19]. BABAR reported good agreement with the $\tau$ data in the most important two-pion channel [20]. The remaining discrepancy with the older $e^{+} e^{-}$ and $\tau$ datasets may be indicative of problems with one or both data sets. It may also suggest the need for additional isospin-violating corrections to the $\tau$ data. Several evaluations of $a_{\mu}^{\mathrm{Had}}[\mathrm{LO}]$ have been published leading to similar results (see Fig. 2). The low-energy contribution to $a_{\mu}^{\mathrm{Had}}[\mathrm{LO}]$ has also been evaluated with the use of additional theory or model constraints in Refs. [22] and [23], respectively.

Higher order, $\mathcal{O}\left(\alpha^{3}\right)$, hadronic contributions are obtained from dispersion relations using the same $e^{+} e^{-} \rightarrow$ hadrons data $[18,21,24]$, giving $a_{\mu}^{\text {Had,Disp }}[\mathrm{NLO}]=(-98.4 \pm 0.6) \times 10^{-11}$, along with model-dependent estimates of the hadronic lightby-light scattering contribution, $a_{\mu}^{\mathrm{Had}, \mathrm{LBL}}[\mathrm{NLO}]$, motivated by large- $N_{C}$ QCD [25-31]. ${ }^{4}$ Following [29], one finds for the sum of the two terms

$$
a_{\mu}^{\mathrm{Had}}[\mathrm{NLO}]=7(26) \times 10^{-11},
$$

where the error is dominated by hadronic light-by-light uncertainties.

Adding Eqs. (6), (9), (11) and (13) gives the representative $e^{+} e^{-}$data based SM prediction

$$
a_{\mu}^{\mathrm{SM}}=116591803(1)(42)(26) \times 10^{-11},
$$

where the errors are due to the electroweak, lowest-order hadronic, and higher-order hadronic contributions, respectively. The difference between experiment and theory

$$
\Delta a_{\mu}=a_{\mu}^{\exp }-a_{\mu}^{\mathrm{SM}}=288(63)(49) \times 10^{-11},
$$

4 Some representative recent estimates of the hadronic lightby-light scattering contribution, $a_{\mu}^{\mathrm{Had}, \mathrm{LBL}}[\mathrm{NLO}]$, that followed after the sign correction of [27], are: $105(26) \times 10^{-11}$ [29], $110(40) \times 10^{-11}[25], 136(25) \times 10^{-11}[26]$. 


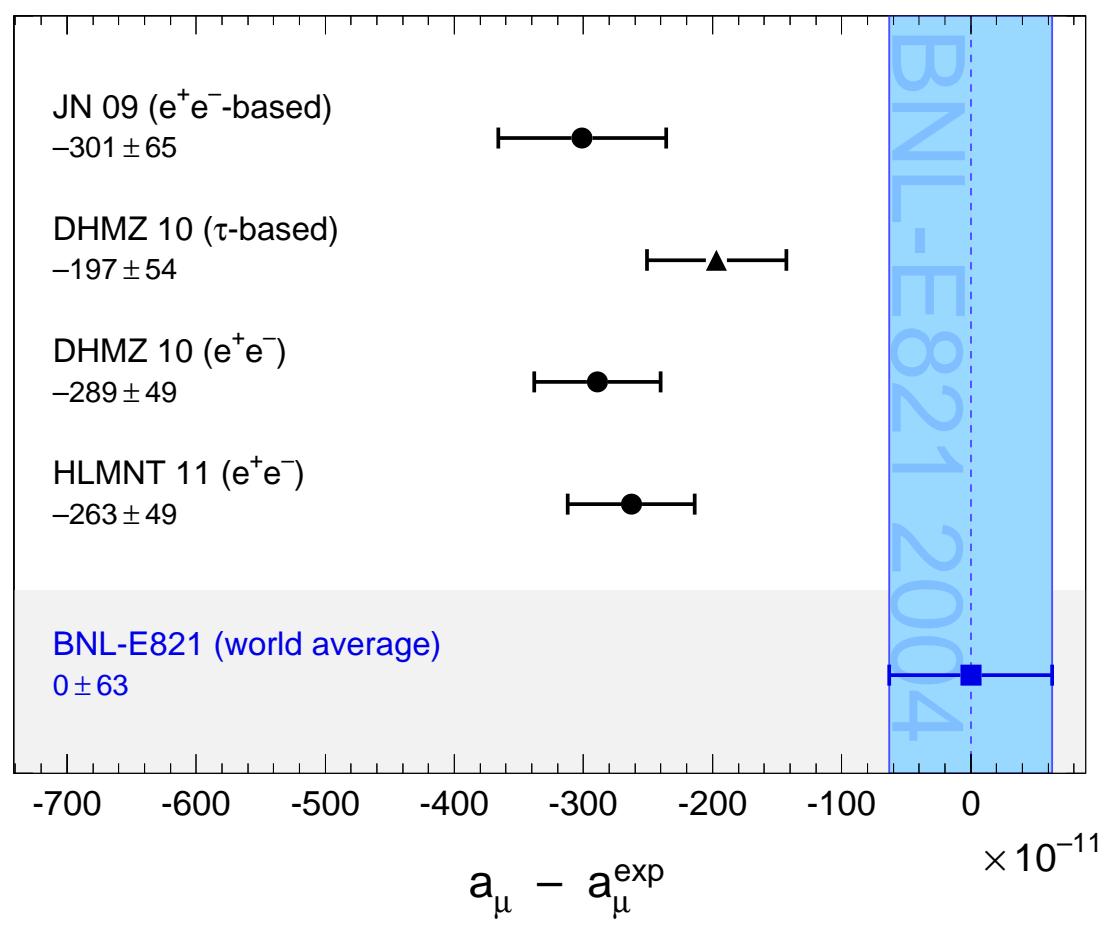

Figure 2: Compilation of recent published results for $a_{\mu}$ (in units of $10^{-11}$ ), subtracted by the central value of the experimental average (3). The shaded band indicates the size of the experimental uncertainty. The SM predictions are taken from: JN [4], DHMZ [17], HMNT [21]. Note that the quoted errors in the figure do not include the uncertainty on the subtracted experimental value. To obtain for each theory calculation a result equivalent to Eq. (15), the errors from theory and experiment must be added in quadrature.

(with all errors combined in quadrature) represents an interesting but not yet conclusive discrepancy of 3.6 times the estimated $1 \sigma$ error. All the recent estimates for the hadronic contribution compiled in Fig. 2 exhibit similar discrepancies. Switching to $\tau$ data reduces the discrepancy to $2.4 \sigma$, assuming the isospin-violating corrections are under control within the estimated uncertainties (see Ref. 32 for an analysis leading to a different conclusion).

An alternate interpretation is that $\Delta a_{\mu}$ may be a new physics signal with supersymmetric particle loops as the leading candidate explanation. Such a scenario is quite natural, since 
generically, supersymmetric models predict [1] an additional contribution to $a_{\mu}^{\mathrm{SM}}$

$$
a_{\mu}^{\mathrm{SUSY}} \simeq \operatorname{sign}(\mu) \cdot 130 \times 10^{-11} \cdot\left(\frac{100 \mathrm{GeV}}{m_{\mathrm{SUSY}}}\right)^{2} \tan \beta,
$$

where $m_{\mathrm{SUSY}}$ is a representative supersymmetric mass scale, $\tan \beta \simeq 3-40$ a potential enhancement factor, and $\operatorname{sign}(\mu)= \pm 1$. Supersymmetric particles in the mass range 100-500 GeV could be the source of the deviation $\Delta a_{\mu}$. If so, those particles should be directly observed at the Large Hadron Collider at CERN.

New physics effects [1] other than supersymmetry could also explain a non-vanishing $\Delta a_{\mu}$. A recent popular scenario involves the "dark photon", a relatively light hypothetical vector boson from the dark matter sector that couples to our world of particle physics through mixing with the ordinary photon [33-35]. As a result, it couples to ordinary charged particles with strength $\varepsilon \cdot e$ and gives rise to an additional muon anomalous magnetic moment contribution

$$
a_{\mu}^{\text {dark photon }}=\frac{\alpha}{2 \pi} \varepsilon^{2} F\left(m_{V} / m_{\mu}\right),
$$

where $F(x)=\int_{0}^{1} 2 z(1-z)^{2} /\left[(1-z)^{2}+x^{2} z\right] d z$. For values of $\varepsilon \sim 1-2 \cdot 10^{-3}$ and $m_{V} \sim 10-100 \mathrm{MeV}$, the dark photon, which was originally motivated by cosmology, can provide a viable solution to the muon $g-2$ discrepancy. Searches for the dark photon in that mass range are currently underway at Jefferson Lab, USA, and MAMI in Mainz, Germany.

\section{References}

1. A. Czarnecki and W.J. Marciano, Phys. Rev. D64, 013014 (2001).

2. M. Davier and W.J. Marciano, Ann. Rev. Nucl. and Part. Sci. 54, 115 (2004).

3. J. Miller, E. de Rafael, and B. Lee Roberts, Rept. Prog. Phys. 70, 795 (2007).

4. F. Jegerlehner and A. Nyffeler, Phys. Reports 477, 1 (2009).

5. J.P. Miller et al., Ann. Rev. Nucl. and Part. Sci. 62, 237 (2012). 
6. P.J. Mohr, B.N. Taylor, and D.B. Newell, CODATA Group, Rev. Mod. Phys. 84, 1527 (2012).

7. G.W. Bennett et al., Phys. Rev. Lett. 89, 101804 (2002);

Erratum ibid. Phys. Rev. Lett. 89, 129903 (2002);

G.W. Bennett et al., Phys. Rev. Lett. 92, 161802 (2004);

G.W. Bennett et al., Phys. Rev. D73, 072003 (2006).

8. J. Bailey et al., Nucl. Phys. B150, 1 (1979).

9. T. Aoyama et al., Phys. Rev. Lett. 109, 111808 (2012);

T. Aoyama et al., Phys. Rev. Lett. 109, 111807 (2012);

T. Kinoshita and M. Nio, Phys. Rev. D73, 013003 (2006);

T. Aoyama et al., Phys. Rev. Lett. 99, 110406 (2007);

T. Kinoshita and M. Nio, Phys. Rev. D70, 113001 (2004);

T. Kinoshita, Nucl. Phys. B144, 206 (2005)(Proc. Supp.);

T. Kinoshita and M. Nio, Phys. Rev. D73, 053007 (2006);

A.L. Kataev, arXiv:hep-ph/0602098 (2006);

M. Passera, J. Phys. G31, 75 (2005).

10. G. Gabrielse et al., Phys. Rev. Lett. 97, 030802 (2006);

Erratum ibid. Phys. Rev. Lett. 99, 039902 (2007);

D. Hanneke, S. Fogwell, and G. Gabrielse, Phys. Rev. Lett. 100, 120801 (2008).

11. R. Bouchendira et al., Phys. Rev. Lett. 106, 080801 (2011).

12. R. Jackiw and S. Weinberg, Phys. Rev. D5, 2396 (1972);

G. Altarelli et al., Phys. Lett. B40, 415 (1972);

I. Bars and M. Yoshimura, Phys. Rev. D6, 374 (1972);

K. Fujikawa, B.W. Lee, and A.I. Sanda, Phys. Rev. D6, 2923 (1972).

13. C. Gnendiger, D. Stöckinger, H. Stöckinger-Kim, Phys. Rev. D88, 053005 (2013);

A. Czarnecki et al., Phys. Rev. D67, 073006 (2003), Erratum ibid. Phys. Rev. D73, 119901 (2006);

S. Heinemeyer, D. Stockinger, and G. Weiglein, Nucl. Phys. B699, 103 (2004);

T. Gribouk and A. Czarnecki, Phys. Rev. D72, 053016 (2005);

A. Czarnecki, B. Krause, and W.J. Marciano, Phys. Rev. Lett. 76, 3267 (1996);

A. Czarnecki, B. Krause, and W.J. Marciano, Phys. Rev. D52, 2619, (1995);

S. Peris, M. Perrottet, and E. de Rafael, Phys. Lett. B355, 523 (1995);

T. Kukhto et al, Nucl. Phys. B371, 567 (1992).

14. G. Degrassi and G.F. Giudice, Phys. Rev. D58, 053007 (1998). 
15. C. Bouchiat and L. Michel, J. Phys. Radium 22, 121 (1961);

M. Gourdin and E. de Rafael, Nucl. Phys. B10, 667 (1969).

16. S.J. Brodsky and E. de Rafael, Phys. Rev. 168, 1620 (1968).

17. M. Davier et al., Eur. Phys. J. C71, 1515 (2011).

18. R. Alemany et al., Eur. Phys. J. C2, 123 (1998).

19. M. Davier et al., Eur. Phys. J. C66, 127 (2010).

20. BABAR Collaboration (B. Aubert et al.), Phys. Rev. Lett. 103, 231801 (2009).

21. K. Hagiwara et al., JPHGB G38, 085003 (2011).

22. S. Bodenstein et al., Phys. Rev. D88, 014005 (2013).

23. M. Benayoun et al., Eur. Phys. J. C73, 2453 (2013).

24. B.Krause, Phys. Lett. B390, 392 (1997).

25. J. Bijnens and J. Prades, Mod. Phys. Lett. A22, 767 (2007).

26. K. Melnikov and A. Vainshtein, Phys. Rev. D70, 113006 (2004).

27. M. Knecht and A. Nyffeler, Phys. Rev. D65, 073034 (2002);

M. Knecht et al., Phys. Rev. Lett. 88, 071802 (2002).

28. J. Bijnens et al., Nucl. Phys. B626, 410 (2002).

29. J. Prades, E. de Rafael, and A. Vainshtein, Advanced series on directions in high energy physics 20, Editors B.L. Roberts and W. Marciano, arXiv:0901.0306 [hep-ph] (2009).

30. J. Hayakawa and T. Kinoshita, Erratum Phys. Rev. D66, 019902 (2002).

31. E. de Rafael, Phys. Lett. B322, 239 (1994).

32. F. Jegerlehner and R. Szafron, Eur. Phys. J. C71, 1632 (2011).

33. P. Fayet, Phys. Rev. D75, 115017 (2007).

34. M. Pospelov, Phys. Rev. D80, 095002 (2009).

35. D. Tucker-Smith and I. Yavin, Phys. Rev. D83, 101702 (R)(2011). 